\title{
Event studies with daily stock returns in Stata - Which command to use?
}

Thomas Kaspereit

\section{UNIVERSITY OF LUXEMBOURG}

30 July 2020 
(1) What are event studies?

(2) What should event study commands be able to do?

(3) Which commands are available?

4. How do these commands compare to each other?

(5) Concluding remarks 


\section{(1) What are event studies?}

(2) What should event study commands be able to do?

Which commands are available?

How do these commands compare to each other?

Concluding remarks 
Event studies measure stock prices reactions to news.

MacKinlay: Event Studies in Economics and Finance

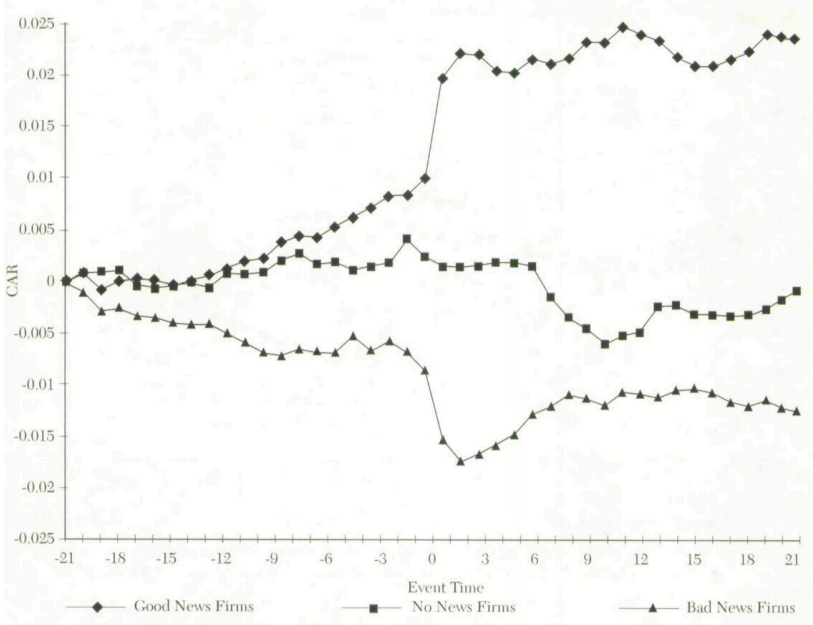


Event studies measure stock prices reactions to news.

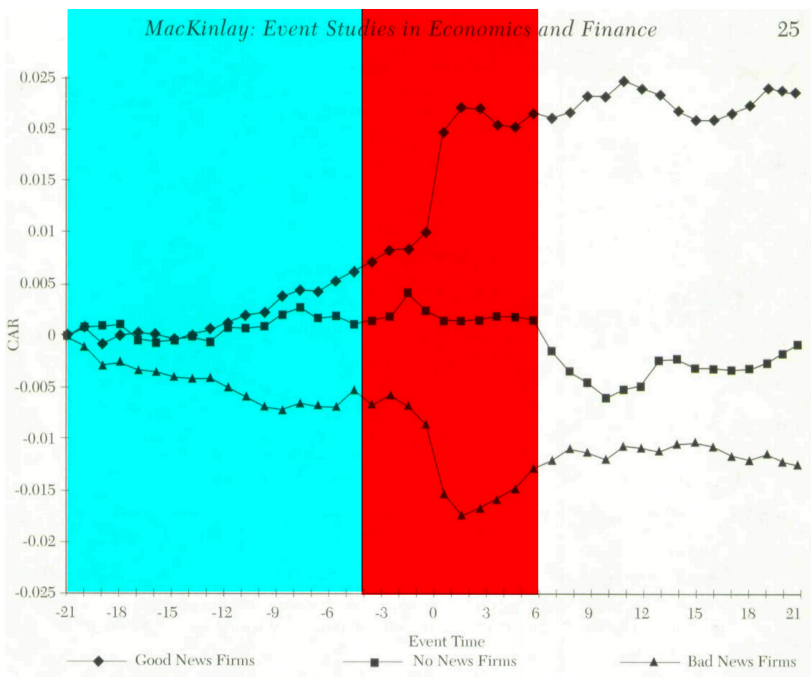


Event studies measure stock prices reactions to news.

\section{Problem:}

Every day, security prices and returns are influenced by many other news events than the event of research interest. 
Event studies measure stock prices reactions to news.

\section{Problem:}

Every day, security prices and returns are influenced by many other news events than the event of research interest.

Solution:

Event studies assume that all others news effects cancel out, except the macro-economic news that affect securities. 


\section{Event studies measure stock prices reactions to news.}

\section{Problem:}

Every day, security prices and returns are influenced by many other news events than the event of research interest.

Solution:

Event studies assume that all others news effects cancel out, except the macro-economic news that affect securities.

Implementation of solution:

Estimation of a (market) index model during the estimation period.

$$
R_{i t}=\alpha_{i}+\beta_{i} R_{m t}+\epsilon_{i}
$$


- Is accounting information decision useful?

- Do changes in dividend policy affects shareholder value?

- Do mergers and acquisitions create or destroy shareholder value?

- Do CEO changes/deaths affect share prices?

- Do announcements of adhering to sustainability principles create shareholder value?

- Do Covid 19 news affect stock prices? 
What are event studies?

(2) What should event study commands be able to do?

Which commands are available?

How do these commands compare to each other?

Concluding remarks 
Event study commands should have at least have the following features:

1. Data management (transforming calendar time to event time)

2. Calculation of average abnormal returns (market index and other models)

3. Assessment of statistical significance of average abnormal returns.

4. Generating output (abnormal return graph, result tables, etc.) 
Event study commands should have at least have the following features:

1. Data management (transforming calendar time to event time)

2. Calculation of average abnormal returns (market index and other models)

3. Assessment of statistical significance of average abnormal returns.

4. Generating output (abnormal return graph, result tables, etc.)
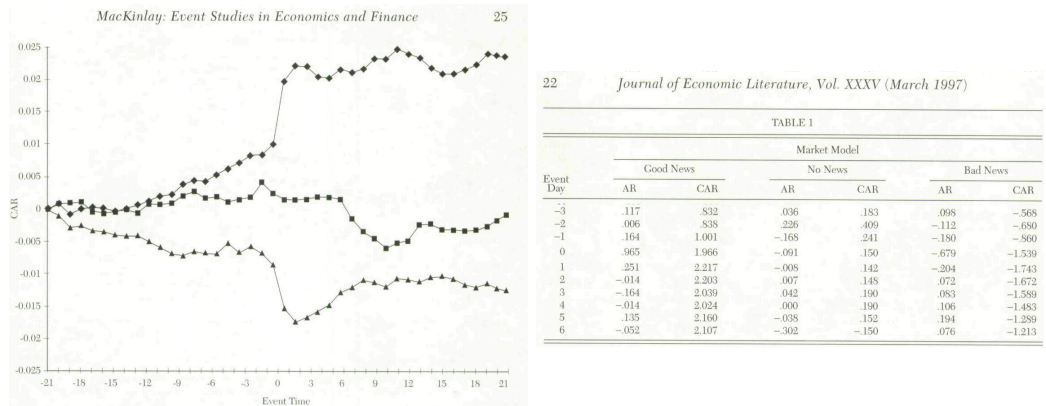
What are event studies?

What should event study commands be able to do?

(3) Which commands are available?

How do these commands compare to each other?

Concluding remarks 
There are currently three user-written commands:

- eventstudy (Zhang et al. 2013)

- eventstudy2 (Kaspereit 2015, 2020)

- estudy (Pacicco et al. 2017, 2020) 
What are event studies?

What should event study commands be able to do?

Which commands are available?

4 How do these commands compare to each other?

Concluding remarks 


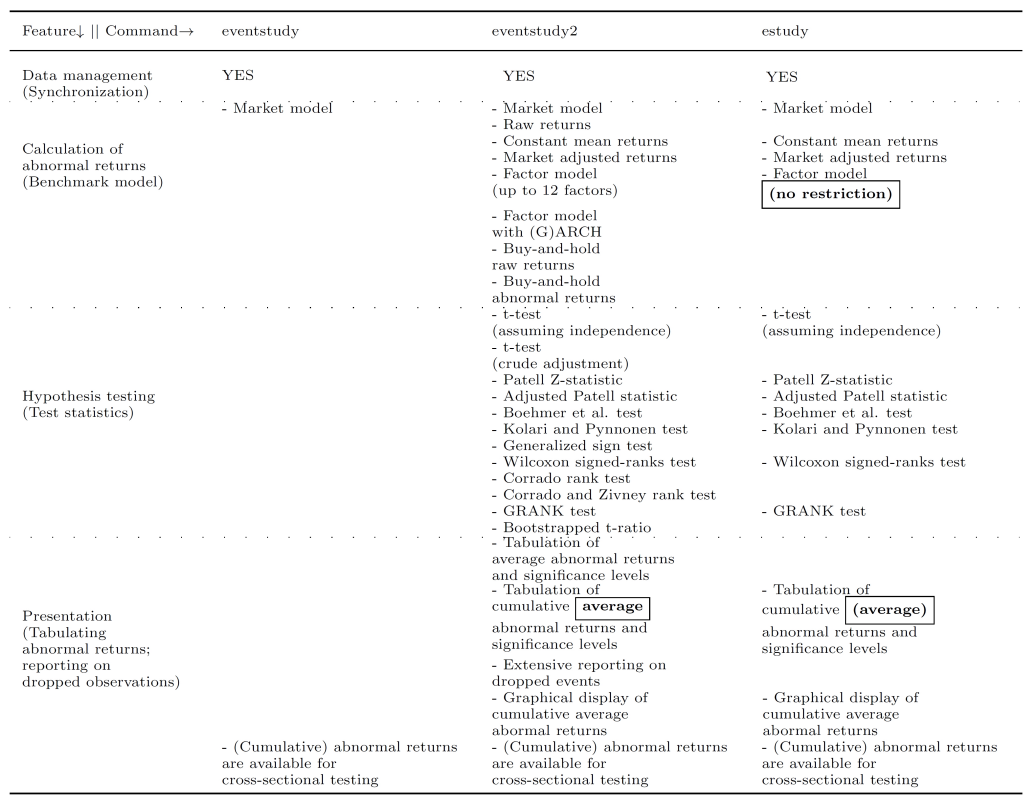




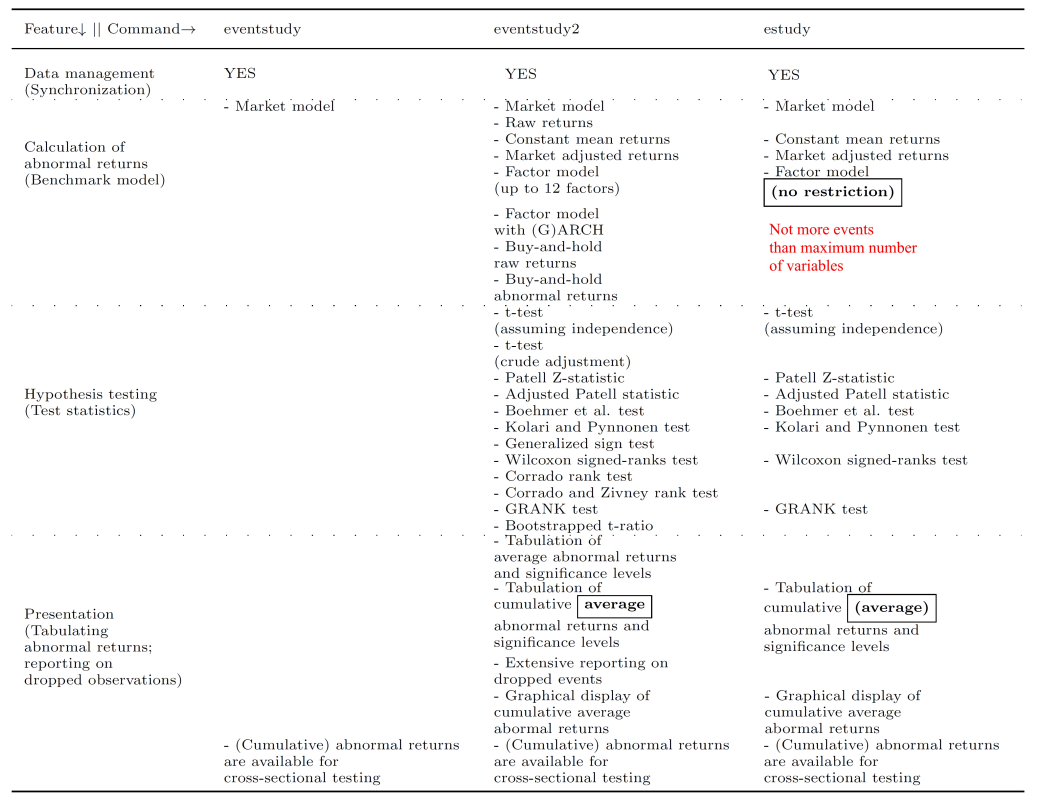


Literature screening of three leading field journals:

- Journal of Accounting Research

- Journal of Finance

- Management Science

Selection and analysis of all 180 event studies that appeared in these journals during the period 2009-2018. 


\begin{tabular}{|c|c|c|c|c|c|}
\hline Authors & Sample period & Datasources & Benchmark models & Test statistics & Events \\
\hline Abarbanell and Park (2017) & $1993-2012$ & CRSP & BH_MATCH & t-Stat & 47,977 \\
\hline Abrahamson et al. (2011) & $1998-2007$ & CRSP & MA & None & 2,788 \\
\hline Agarwal et al. (2013) & $2004-2007$ & CRSP & MM & Patell Z, GenSign & 66 \\
\hline Agarwal et al. (2016) & $1998-2010$ & CRSP & MA & None & 3,046 \\
\hline Aggarwal et al. (2015) & $2007-2009$ & CRSP & RAW & None & 3,053 \\
\hline kenneth R. Ahern and Harford (2014) & $1986-2010$ & CRSP & MA & None & not reported \\
\hline Akbas (2016) & $1980-2011$ & CRSP & MA & None & 366,454 \\
\hline Albuquerque and Schroth (2015) & $1990-2010$ & CRSP & RAW & None & 114 \\
\hline Allee and DeAngelis (2015) & $2004-2014$ & CRSP & $\mathrm{MA}, \mathrm{FM}$ & None & 33,428 \\
\hline Ammann et al. (2016) & $1992-2008$ & CRSP & MM & t-Stat & 1,875 \\
\hline Anderson et al. (2012) & $2005-2007$ & CRSP & PEA & t-Stat & 1,571 \\
\hline Anderson et al. (2018) & $1992-2014$ & CRSP & RAW & None & 27,615 \\
\hline Arikan and Stulz (2016) & $1975-2008$ & CRSP & MA & t-Stat, Wilcoxon & 3,081 \\
\hline Ashbaugh-Skaife et al. (2009) & $2003-2005$ & CRSP & BH_IND & Wilcoxon & 787 \\
\hline Babenko (2009) & $1996-2002$ & CRSP & MM & None & 1,174 \\
\hline Badoer and James (2016) & $2001-2001$ & CRSP Treasury & COMEAN & t-Stat & 1 \\
\hline Becher et al. (2015) & $1993-2008$ & CRSP & RAW, CAL & None, t-Stat & 5,381 \\
\hline Berkman and Truong (2009) & $2000-2004$ & CRSP, yahoo!Finance & $\mathrm{BA}$ & t-Stat & 38,031 \\
\hline Berkman et al. (2014) & $1999-2010$ & Compustat Global & MA & t-Stat & 4,136 \\
\hline Bernhardt et al. (2016) & $2003-2010$ & CRSP & BH_IND & t-Stat & 24,793 \\
\hline Betton et al. (2014) & $1980-2008$ & CRSP & MM & None & 6,150 \\
\hline Bhojraj et al. (2009) & $1988-2006$ & CRSP & MA, BH_MATCH, CAL & t-Stat, BS t-Stat & 35,530 \\
\hline Blankespoor et al. (2017) & $2011-2013$ & CRSP & BH_MATCH & None & 224 \\
\hline Bradley et al. (2017) & $1983-2011$ & CRSP & $\mathrm{MA}$ & None & 40,719 \\
\hline Brennan et al. (2016) & $1983-2010$ & CRSP & $\mathrm{MA}$ & None & not reported \\
\hline Brown and Tucker (2011) & $1997-2006$ & CRSP & MA & None & 23,487 \\
\hline Bruno et al. (2016) & $1999-2003$ & CRSP & BH_MATCH & t-Stat & 2,002 \\
\hline Bushee et al. (2010) & $1993-2004$ & CRSP & MA & None & 27,987 \\
\hline Bushee et al. (2011) & $1999-2007$ & CRSP & BH_MATCH & t-Stat, Wilcoxon & 95,105 \\
\hline Bushman et al. (2017) & $2000-2012$ & CRSP & $\mathrm{MA}$ & None & 41,760 \\
\hline Call et al. (2018) & $1978-2012$ & CRSP & MA & None & 658 \\
\hline Cao and Narayanamoorthy (2012) & $1987-2008$ & CRSP & BH_IND & None & 305,908 \\
\hline Cao et al. (2015) & $2000-2010$ & CRSP & BH_IND, PEA & None & 40,807 \\
\hline Cen et al. (2016) & $1979-1995$ & CRSP & BH_MATCH & None & 62,041 \\
\hline Chang et al. (2010) & $1992-2002$ & CRSP & MM, FM, BH_IND, BH_MATCH & t-Stat, Wilcoxon & 298 \\
\hline Chava et al. (2018) & $1989-2007$ & CRSP & MM & None & 1,677 \\
\hline Cheong and Thomas (2018) & $1993-2013$ & CRSP & MA & None & 197.004 \\
\hline Chhaochharia et al. (2017) & $1999-2006$ & CRSP & MM & None & 6,643 \\
\hline Choudhary et al. (2009) & $2004-2005$ & CRSP & MM & t-Stat & 365 \\
\hline Christensen et al. (2009) & $2004-2004$ & CRSP & MA & None & 136 \\
\hline Cohen and Schmidt (2009) & $\begin{array}{l}2004-2004 \\
1993-2003\end{array}$ & CRSP & MA & None & 266,520 \\
\hline Cohn et al. (2016) & $2010-2010$ & CRSP & COMEAN & $\mathrm{CDA}$ & $\begin{array}{rl}3 & 0,0,0 \\
3\end{array}$ \\
\hline Collin-Dufresne and Fos (2015) & $1994-2010$ & CRSP & BH_IND & t-Stat & 3.126 \\
\hline Crane and Koch (2018) & $1980-2012$ & CRSP & MA & None & 26,766 \\
\hline
\end{tabular}


Panel A: All three journals

Fully applicable

Partially applicable 4

$5 \quad 8.33 \%$

Not applicable

161

$2.22 \%$

$89.44 \%$

$\begin{array}{rr}163 & 90.56 \% \\ 5 & 2.78 \% \\ 12 & 6.67 \%\end{array}$

96

$53.33 \%$

$17 \quad 9.44 \%$

$67 \quad 37.22 \%$

Panel B: Journal of Accounting Research

Fully applicable $\quad 3 \quad 5.45 \%$

Partially applicable $\quad 1 \quad 1.82 \%$

Not applicable

51

$92.73 \%$

$\begin{array}{rr}54 & 98.18 \% \\ 0 & 0.00 \% \\ 1 & 1.82 \%\end{array}$

$56.36 \%$

$9.09 \%$

$\begin{array}{rr}5 & 9.09 \% \\ 19 & 34.55 \%\end{array}$

Panel C: Journal of Finance

Fully applicable

$\begin{array}{rr}6 & 8.45 \% \\ 2 & 2.82 \% \\ 63 & 88.73 \%\end{array}$

$\begin{array}{rr}65 & 91.55 \% \\ 1 & 1.41 \% \\ 5 & 7.04 \%\end{array}$

$\begin{array}{rr}41 & 57.75 \% \\ 4 & 5.63 \% \\ 26 & 36.62 \%\end{array}$

Not applicable

$88.73 \%$

Panel D: Management Science

\begin{tabular}{lrrrrrr} 
Fully applicable & 6 & $11.11 \%$ & 44 & $81.48 \%$ & 24 & $44.44 \%$ \\
Partially applicable & 1 & $1.85 \%$ & 4 & $5.63 \%$ & 8 & $14.81 \%$ \\
Not applicable & 47 & $87.04 \%$ & 6 & $8.45 \%$ & 22 & $40.74 \%$ \\
\hline
\end{tabular}



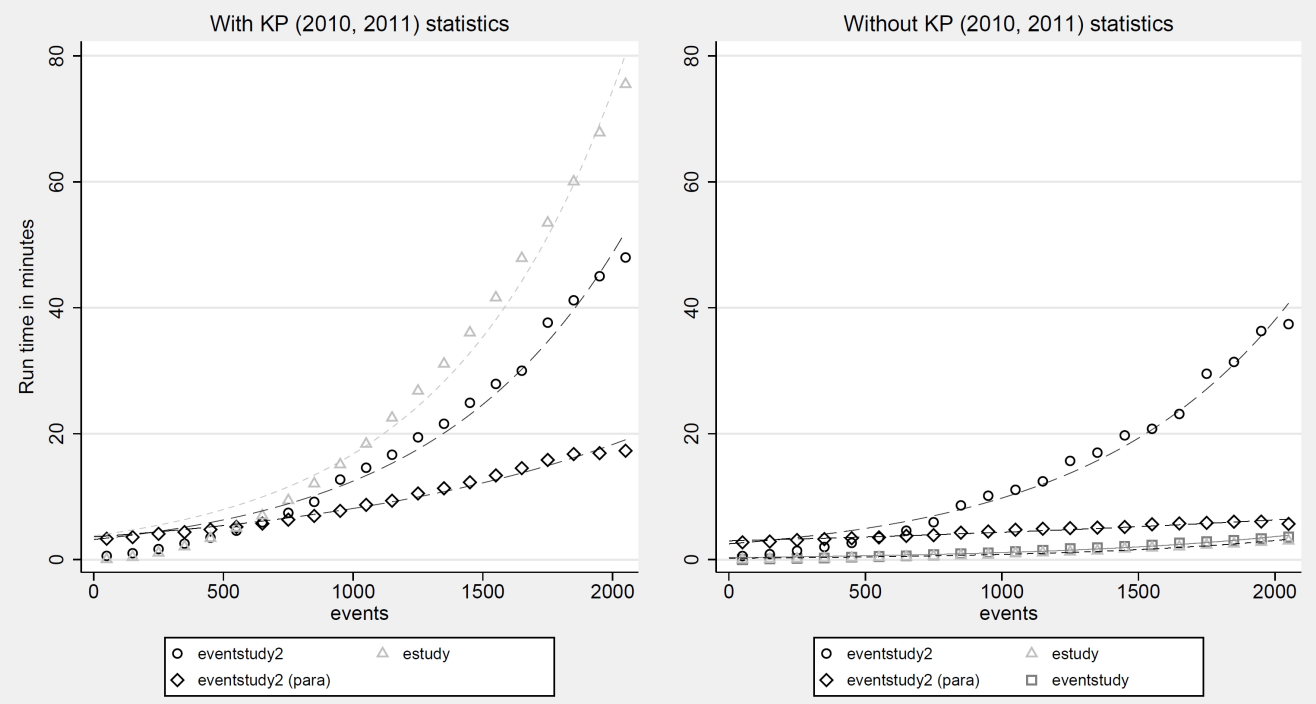
What are event studies?

(2) What should event study commands be able to do?

Which commands are available?

How do these commands compare to each other?

\section{(5) Concluding remarks}


The three command do not represent evolutions but can be best choices in different scenarios:

- eventstudy: simple studies, Stata beginners, assessment of statistical significance not required

- eventstudy2: very complex and large studies, extremely accurate results

- estudy: complexer studies of limited size ( $<24,000$ events), comfortable output (LaTeX), by event assessment of statistical significance 
Many thanks to...

- Bill Rising for setting up and testing my Zoom connection.

- the Stata Corp. crew for organizing the conference.

- Joe Newton for providing valuable input during the ongoing review process in the Stata Journal. 